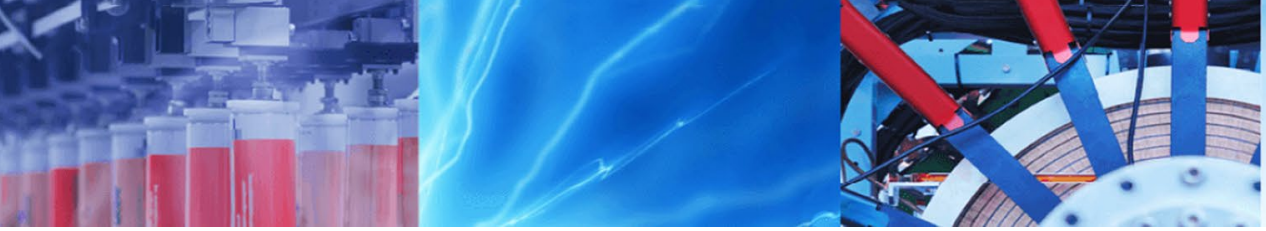

Short Communication

\title{
A study on buckling of simply supported plates with edges partially restrained for in-plane movement
}

\author{
Kanta Prajapat $^{1}$ (D) Samit Ray-Chaudhuri ${ }^{2} \cdot$ Ashwini Kumar $^{3}$
}

Received: 31 October 2019 / Accepted: 23 October 2020 / Published online: 9 November 2020

(c) Springer Nature Switzerland AG 2020

\begin{abstract}
This paper investigated experimentally the influence of size and location of a circular cut-out on the buckling load of simply supported plates with in-plane boundary conditions (and thus, induced stress pattern in the pre-buckling state). Experiments were conducted on solid and perforated stainless steel plates with different aspect ratios and edges partially restrained for in-plane movements. Results of these experiments demonstrated that the size and position of cut-out significantly affected the buckling load of the plate specimens. Further, to better interpret the experimentally obtained results, finite element analyses were conducted wherein the numerical model closely represented the experimental setup. The Riks method of analysis and eigenvalue elastic buckling analysis were carried out with the same set of experimental boundary conditions. From these analysis, it was found that the in-plane restraints significantly affected the buckling behavior of a plate. It was also observed that the buckling loads obtained from experiments matched reasonably well with the analytical values.
\end{abstract}

Keywords Plate buckling · Pre-buckling stresses $\cdot$ Experimental investigation · Load-deformation curve · In-plane restraints

\section{Introduction}

For a thin plate, various parameters govern its buckling load. One such important (often critical) parameter is the plate boundary conditions. Numerous experimental and analytical investigations have been carried out so far focusing on the buckling of plates. Many of these have concentrated on the issues of support and loading conditions $[2,3,8,10,12-14,17,18]$. However, not too many studies were conducted on how the in-plane boundary conditions influence the buckling load of a thin plate. For example, along the unloaded edges, Bedair and Sherbourne [5, 6] and Bedair [4] considered the influence of full and partial in-plane normal restraints. Different loading conditions viz. uni-axial compression, bending and shear were considered for this purpose. It was noted from their study that along the unloaded edges, in-plane restraints lowered the buckling load in comparison to the case of without any in-plane restraint. Effect of different in-plane boundary conditions on elastic buckling behavior of solid and perforated plates under edge compression has been recently considered by Prajapat et al. [16].

Many experimental studies have been conducted so far to determine the buckling load for rectangular plates with or without any cut-out under simply supported or clamped conditions. Various experimental set-ups were used for this purpose by different investigators. For brevity, only a few of these studies are discussed here. Dietrich et al. [9] performed an experimental study on plastic buckling of simply supported plates under edge compression.

Kanta Prajapat, prajapat@iastate.edu; kantaiitk@gmail.com; Samit Ray-Chaudhuri, samitrc@iitk.ac.in; Ashwini Kumar, ashwini@iitgn.ac.in | 1 Department of Civil, Construction and Environmental Engineering, lowa State University, Town Engineering Building, 423 Town Engineering, Ames, IA 50011, USA. ${ }^{2}$ Department of Civil Engineering, IIT Kanpur, Kanpur, UP 208016, India. ${ }^{3}$ Civil Engineering, IIT Gandhinagar, Gandhinagar 382355, India. 
On loaded edges, grooved arrangement was used to simulate the simply supported conditions. Whereas, special tube column arrangements with grooved disks were used at the unloaded edges for the same. In some studies, simple' $V$ ' grooved supporting bars or knife edged blocks were also used at the unloaded edges to incorporate the simply supported boundary conditions [11]. A more advanced arrangement was used by Bradfield [7] to simulate various boundary conditions. In this study, discrete finger supports were used on the unloaded edges to simulate rotationally free to rotationally locked edge conditions. The major focus of these studies was placed on out-ofplane boundary conditions without much importance to in-plane restraints on edges.

In practice, various in-plane restraints may arise along the boundaries of a plate due to the connection of the plate with adjacent structural elements. These in-plane restraints cause an in-plane stress distribution, which is quite different than the applied stresses. Hence, studies are needed to account for such situations. This work considered the effect of in-plane edge restraints (perpendicular to the direction of loading) to study the buckling behavior of square and rectangular simply supported plates under uni-axial compression. At first, experiments were performed in laboratory on simply supported solid and perforated stainless steel plates. Different aspect ratios and edges restraint were considered. To confirm the experimental results, a numerical model, which closely represented the experimental setup, was developed in ABAQUS [1], a widely used commercial finite element package. Analysis was performed using Riks method in the framework of ABAQUS. An eigenvalue elastic buckling analysis with the same set of boundary conditions, was also carried out to study how the elastic buckling load compared with the experimental data.

\section{Test set-up, instrumentation and test procedure}

An experimental study on a few thin solid and perforated plate specimens was carried out in this work to experimentally obtain the load-deformation response and buckling strength of these plates. The details of test set-up are shown in Figs. 1 and 2. The set-up consisted of four primary supporting plates (left, right, top and bottom) to hold the specimen and two secondary supporting plates to prevent the outward spreading of the left and right primary supporting plates. To achieve simply supported boundary conditions, a groove was made along the length of the primary supporting plates (the details of groove are given in Fig. 1c). Stainless steel plates $\left(E=1.9 \times 10^{5} \mathrm{MPa}\right.$, yield stress $=250 \mathrm{MPa}, v=0.31$ and thickness $=1.2 \mathrm{~mm}$ ) of three different sizes $\left(176 \times 210,176 \times 93\right.$ and $176 \times 61 \mathrm{~mm}^{2}$, aspect ratios nearly equal to 1,2 and 3 , respectively) were used for the tests. The mechanical properties of the plate specimens (as mentioned here) were adopted from the manufacturer's specification. However, a few coupon tests were also performed and it was noted that the test results were comparable with the manufacturer's specification. Table 1 provides the details of all eight specimens tested in the laboratory. Out of the eight specimens, there were 3 solid plates having aspect ratio (nearly) 1,2 , and 3 . Remaining five specimens were plates with a cut-out (see Table 1 for cut-out size definition). Out of these five specimens three were with cut-out size 0.2 and two with the size 0.5 . In four specimens the cut-out was placed centrally and in only one case it was close to the loaded edge of the plate. Under the limitation of available resources to check any initial imperfection (such as straight edge), no noticeable imperfections were observed in the plate specimens. The specimens were placed in the setup as shown in Fig. 1a. In the bottom supporting plate, two pins, which were projected $1.5 \mathrm{~mm}$ in to the groove depth were used at each end of the plate specimen to prevent the transverse movement of the bottom edge of the plate. Load was applied through a hand driven screw jack fitted with a $100 \mathrm{kN}$ button-type load cell. To avoid any eccentricity in loading and to provide electrical insulation to the load cell, it was fitted in two grooved bakelite plates at the top and bottom. The circular groove in the top face of top bakelite plate was holding the screw jack's head and the grooved bottom face was accommodating the pressure point of circular load cell. In a similar manner, the grooved top face of bottom bakelite plate was accommodating the bottom of circular load cell and was firmly attached to the center of top supporting plate and thus, ensuring no eccentricity in loading (see Fig. 2c). To ensure that the load was being transferred to the plate specimen only and not to the supporting assembly, a nominal gap of $1.5 \mathrm{~mm}$ was kept on each side between the side supporting plate and the top supporting plate. It may be noted that the applied load was thus distributed over a width of $5 \%, 10 \%$ and $16 \%$ smaller than the total width of the plate specimen for aspect ratio 1,2 and 3, respectively. To measure the out-of-plane deflection at the desired points on the plate, linear variable differential transformers (LVDTs) were used. Figure 2 shows the complete test set-up in the laboratory. Figure 3 provides the picture of solid plate specimens of three aspect ratios (see Table 1) used for the experiments. 
Fig. 1 Schematic diagrams are shown for: a test set-up, b primary supporting plate details, c details of groove, and $\mathbf{d}$ loading assembly (front view)

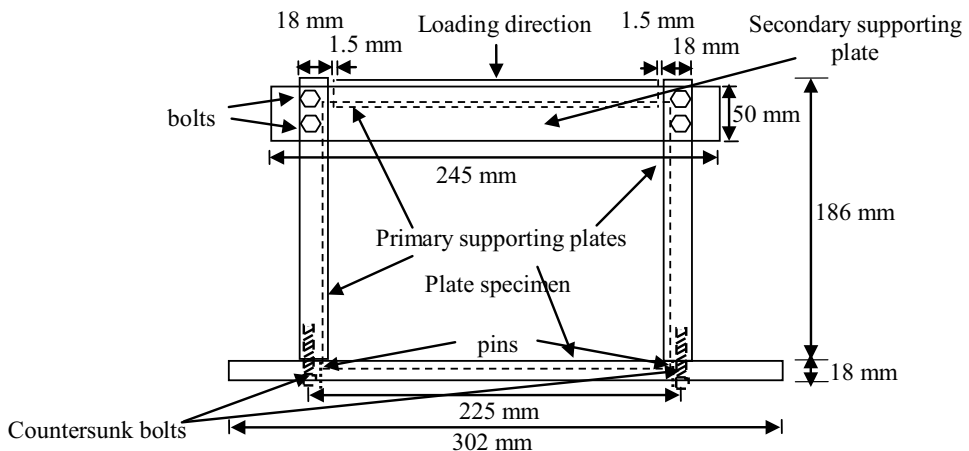

(a)

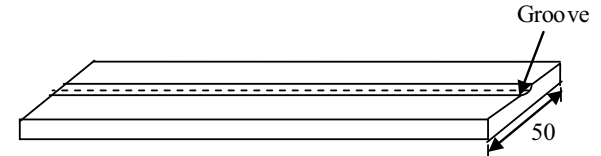

(b)

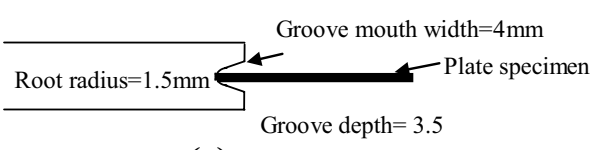

(c)

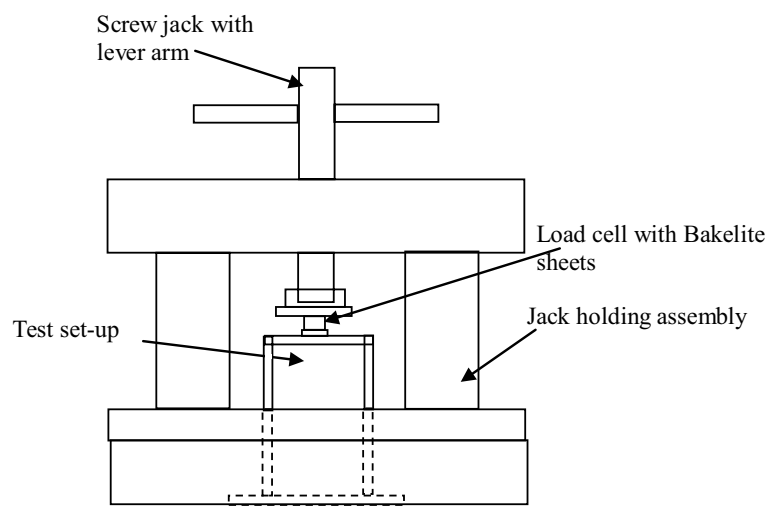

(d)

\section{Experimental results}

Figures 4, 5, 6 and 7 show the experimental load deformation responses for all the plate specimens considered in this study. Although the degradation in stiffness is visible in Figs. 4, 5, 6 and 7, unlike a plate with unsupported unloaded edges, which behaves more like a column, a sudden drop in the load was not observed in these load deformation curves. This is because the plate in the present study was well supported at the unloaded edges (Table 2), and therefore, the load versus out-ofplane deformation response of the plate showed significant strength after the first stiffness degradation point (i.e., the plate specimens showed some post buckling strength). Since the load deformation behavior of plate was not showing any distinct first stiffness degradation point, buckling load in the present study was obtained as the point of intersection of the first tangent and the second tangent of the load deformation curve as shown in Figs. 4, 5, 6 and 7 and Table 3. The logic behind this approach is that, on the commencement of buckling, the stiffness of the plate reduces, which causes a drop in the slope of the load-deformation curve. Table 4 provides the experimentally obtained buckling load for each plate specimen.

Owing to the opacity provided by the supporting assembly, it was difficult to capture a visibly deformed shape of the specimens through pictures. However, for the clarity of presentation, based on close inspection of deformation pattern, a representative schematic diagram along with the picture of a loaded specimen showing the deformed shape are provided in Fig. 8. The specimen shown in Fig. $8 \mathrm{~b}$ was having an aspect ratio nearly equal to one and appeared to be buckled in a single half wave in both the directions. 
Fig. 2 Experimental set-up is shown for: a test set-up with loading assembly, $\mathbf{b}$ picture of experimental set-up showing LVDTs and other details, and $\mathbf{c}$ enlarged screw jack head with load cell and bakelite plates

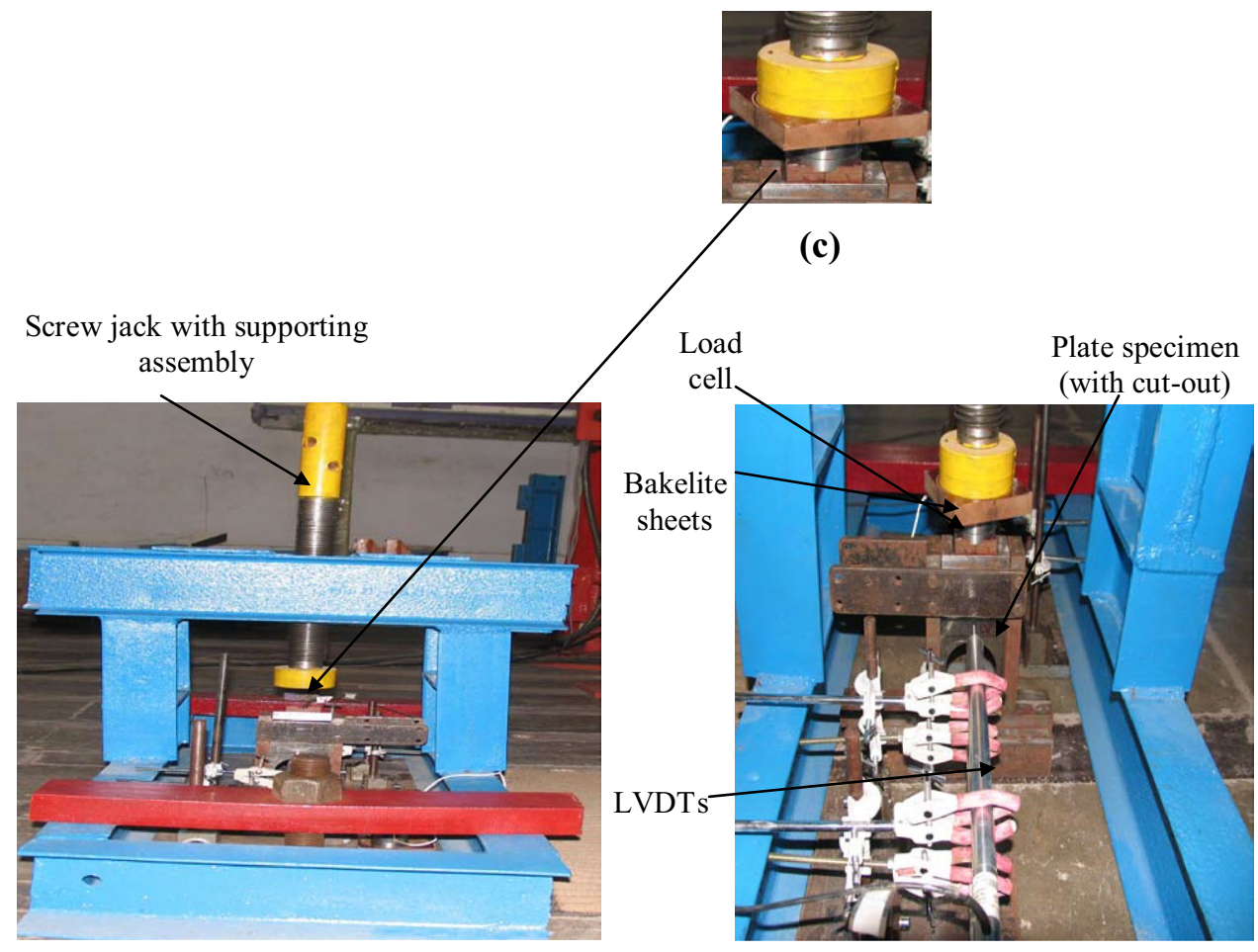

(a) (b)
Fig. 3 Pictures of specimen numbered 1, 4 and 8 (from the left to right) are shown

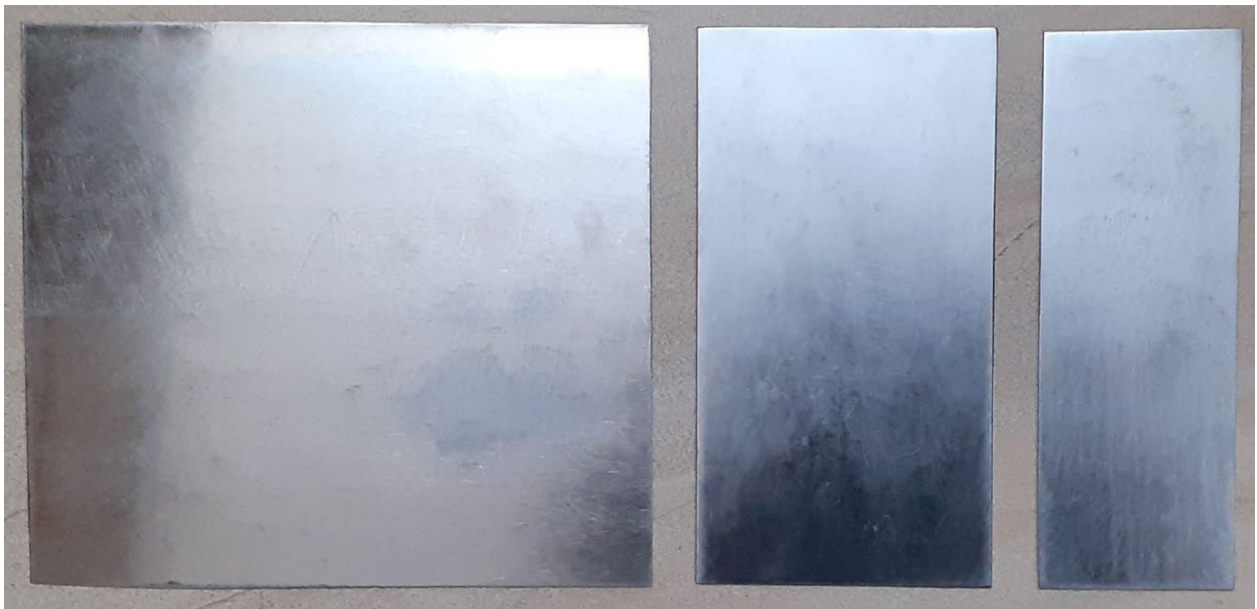

\subsection{Effect of size and position of cut-outs}

For aspect ratio close to unity (Specimens 1,2 and 3), one can note from Table 4 that the presence of cut-out at the center decreases the buckling load. Also, as the cut-out size increases, the buckling load reduces. It may be noted that, for aspect ratio 1 , the plate buckles almost in a single half wave. Thus, the presence of cut-out at the middle makes the plate susceptible to larger out-of-plane deformations, and this susceptibility of out-of-plane movement increases with an increase in cut-out size. Thus, a decrease in elastic buckling load is observed as the cut-out size increases. For cut-out size $d / b=0.5$, the buckling load is about $59 \%$ of that of the unperforated plate specimen.

For aspect ratio close to 2 (Specimens 4, 5, 6 and 7), it may be noted from Table 4 that the buckling load does not change even if a central circular cut-out of size $d / b=0.2$ is introduced (Specimens 4 and 5). The reason behind this is as follows: for small cut-out size, the plate with in-plane restraints buckles in the second mode in the loading direction (i.e., two half-waves) and since the buckling resistance is mainly provided by the central region of two plate panels along the $x$-axis, a small cut-out at the center of the specimen does not 
Table 1 Details of test specimens

\begin{tabular}{llllll}
\hline Specimen no. & Specimen size & Aspect ratio & Cut-out size $d / b$ & Cut-out number and position \\
\hline 1 & $176 \times 210 \times 1.2$ & 0.838 & 0 & 0 \\
2 & $176 \times 210 \times 1.2$ & 0.838 & 0.2 & 1 at centre \\
3 & $176 \times 210 \times 1.2$ & 0.838 & 0.5 & 1 at centre \\
4 & $176 \times 93 \times 1.2$ & 1.892 & 0 & 0 \\
5 & $176 \times 93 \times 1.2$ & 1.892 & 0.2 & 1 at centre \\
6 & $176 \times 93 \times 1.2$ & 1.892 & 0.2 & Right figure \\
7 & $176 \times 93 \times 1.2$ & 1.892 & 0.5 & 1 at centre \\
8 & $176 \times 61 \times 1.2$ & 2.885 & 0 & 0 & \\
\hline
\end{tabular}

result in a decrease in buckling stiffness. But, as the cutout moves towards the center of the adjacent panel, there is a slight decrease in the buckling coefficient (Specimen 6). Similarly, as the cut-out size at the center increases, this affects the stiffness of the buckling resistance providing zone and the susceptibility of out-ofplane movement increases (Specimen 7).

For aspect ratio nearly 3 and for plate specimen with no cut-out (Specimen 8), the buckling load is highest among all the plate specimens considered. This because, with in plane restraints, the plate buckles in the third mode and thus providing higher buckling loads than the other cases considered in this study.

\section{Numerical simulation}

For a plate with length $a$, width $b$, thickness $h$, Young's modulus $E$ and Poisson's ratio $v$ the critical stress can be obtained as:

$\sigma_{c r}=\frac{k D \pi^{2}}{b^{2} h}$

where $D$ is the flexural rigidity of plate given by $E h^{3} / 12\left(1-v^{2}\right)$ and $k$ is a dimensionless buckling load factor of the plate. In achieving Eq. (1), it is assumed that the in-plane normal $\left(\sigma_{x}, \sigma_{y}\right)$ and shear stresses $\left(\tau_{x y}\right)$ at any point in the plate are identical to the employed in-plane stresses. This assumption is true for the plate with no in-plane restraints at plate edges. However, in practice the situation may be different and hence, this assumption can mislead the buckling analysis. Therefore, the exact determination

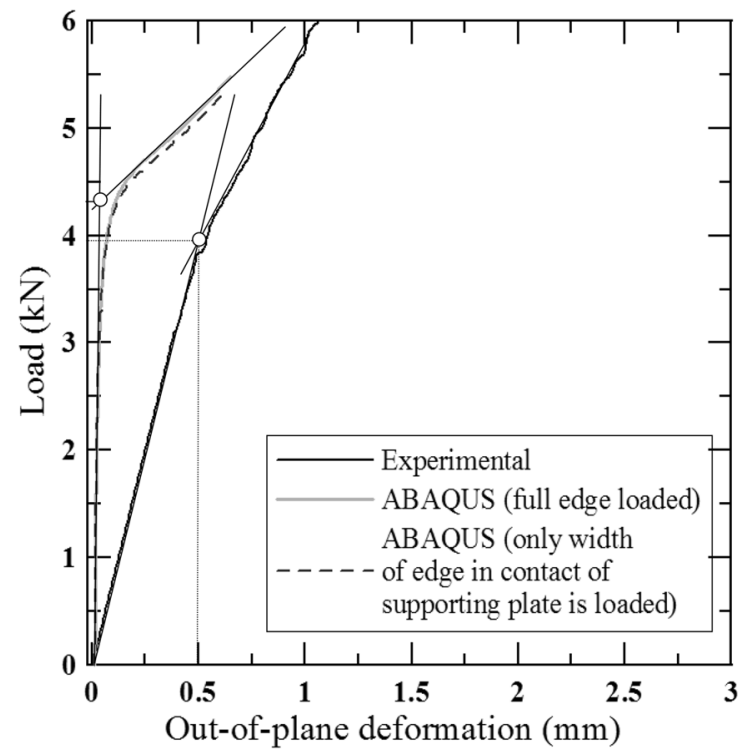

(a) specimen 1

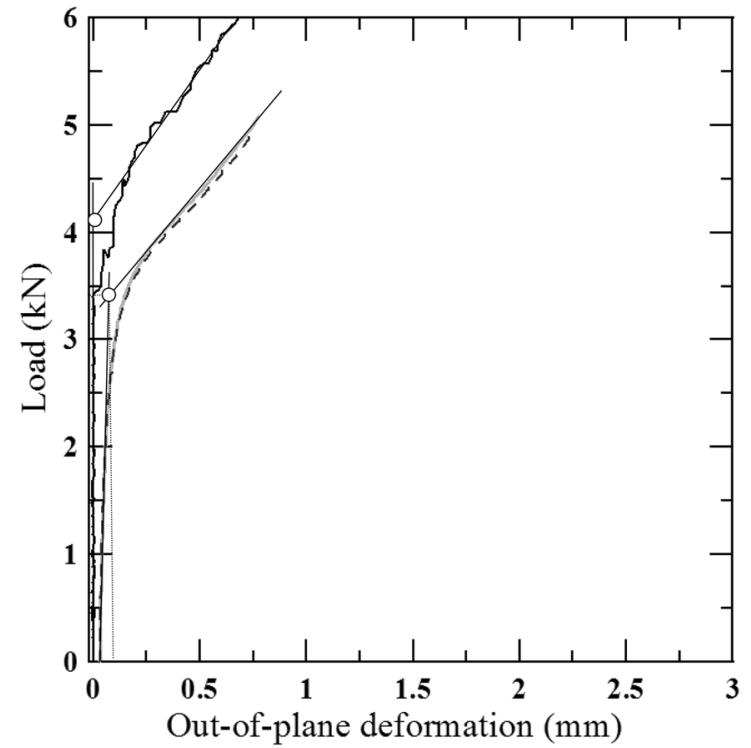

(b) specimen 2

Fig. 4 Plots show load versus out-of-plane deformation 


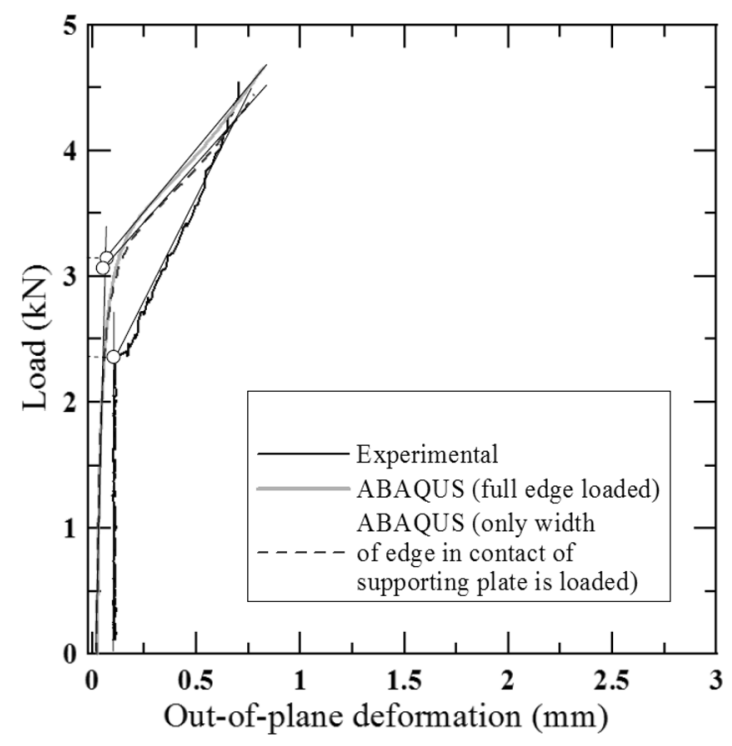

(a) specimen 3

Fig. 5 Plots show load versus out-of-plane deformation

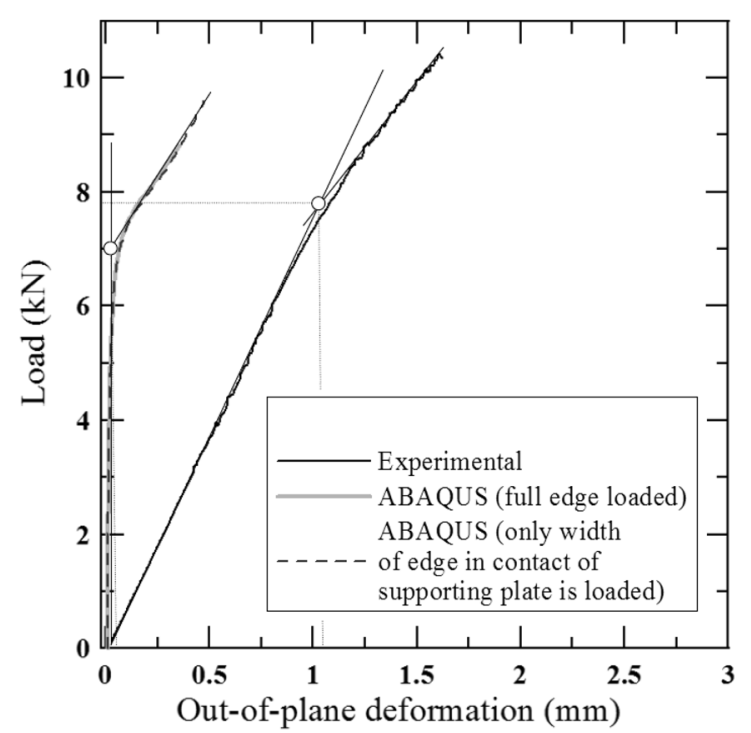

(a) specimen 5

Fig. 6 Plots show load versus out-of-plane deformation

of buckling load of a plate requires determination of these pre-buckling stresses accurately.

The boundary conditions arising from the test setup in this study can be represented as given in Table 2 . It can be observed from this table that the test set-up involves the in-plane restraints along the plate boundaries. Therefore, it is required to consider these restraints and the induced pre-buckling stresses in numerical analysis for an appropriate comparison of experimental and numerical results.

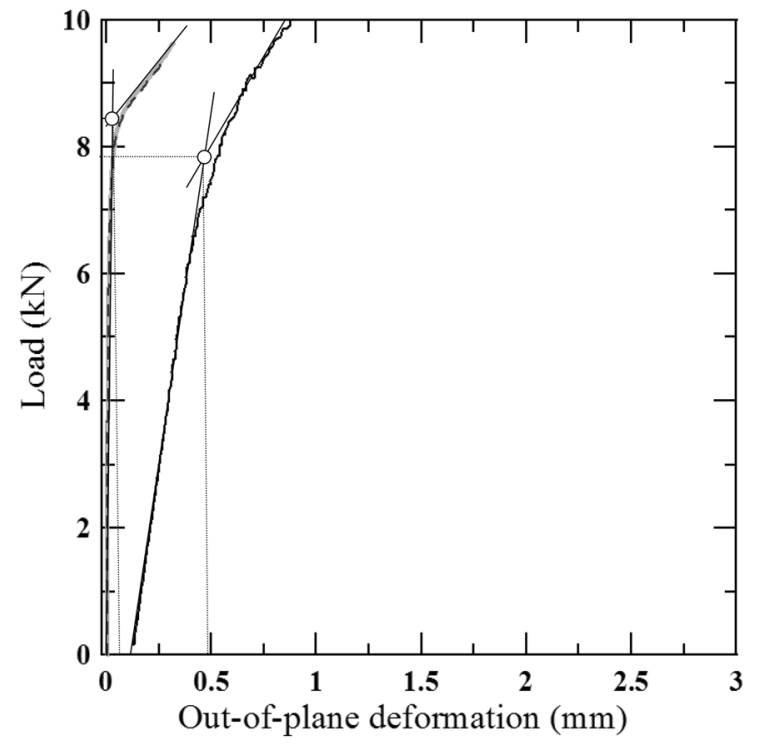

(b) specimen 4

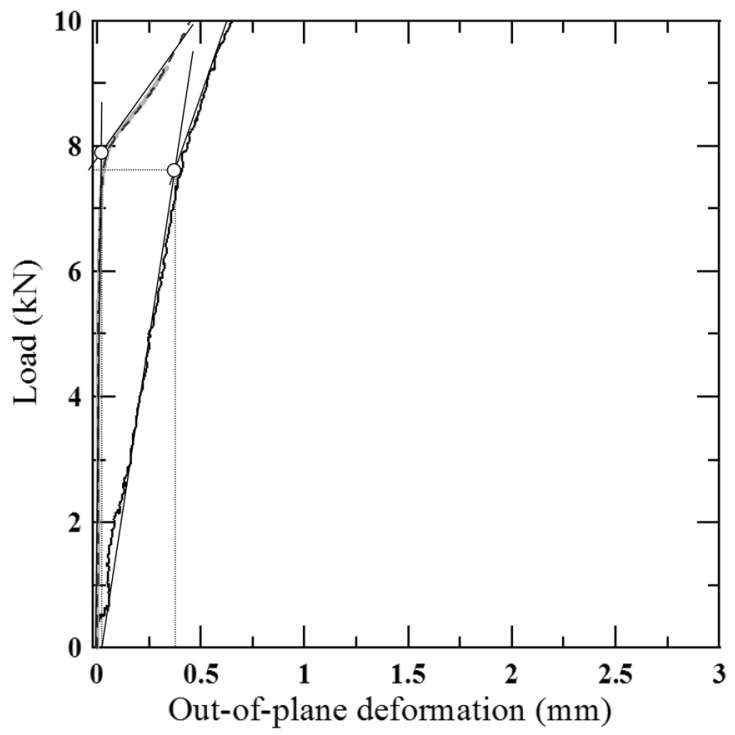

(b) specimen 6

The pre-buckling stresses can be determined by solving the plane elasticity problem using the known force or displacement boundary conditions. In this study, it is incorporated by considering the actual in-plane restraints of plate edges in finite element package Abaqus [1] for determining the buckling behavior of plate numerically.

A numerical model, which closely represents the experimental setup, was developed in Abaqus [1]. Considering the fact that the bottom edge of the plate specimen is 


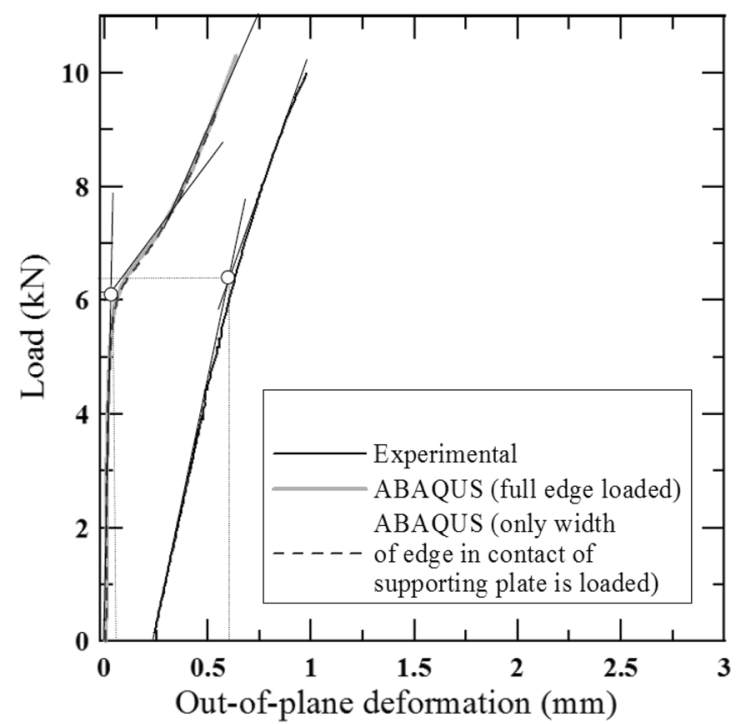

(a) specimen 7

Fig. 7 Plots show load versus out-of-plane deformation

Table 2 In-plane boundary conditions for experimental study

\begin{tabular}{lll}
\hline Loaded edge & Opposite to loaded edge & Side edges \\
\hline$u \neq 0, v=0$ & $u=0, v=0$ & $u \neq 0, v=0$ \\
\hline
\end{tabular}

$u, v=$ displacement along and perpendicular to the direction of loading

supported on a rigid (strong) floor through primary supporting plate, for simplification of analysis, in numerical modeling it is assumed that the supporting assembly generates a uniform load distribution on both loaded edges of the plate. Since the applied load was distributed over a width smaller than the plate specimen (Sect. 2), to study the effect of this reduction of load width, numerical simulations were done for both full edge loading condition and loading over the width of the plate specimen in contact of the top primary supporting plate only.

The optimum mesh detailing (element type and number) was obtained by comparing different elements and their number for their capability to generate the buckling load factor evaluated from the plate buckling theory (details can be found in Prajapat [15]). Based on this comparison, element S8R5 available in ABAQUS standard library, with a fine mesh (element side-length about $b / 40$ ) was adopted for this study due to its computational efficiency and accuracy over other elements. It may be noted that S8R5 is a 8-node thin shell element, which is doubly curved with 5 degrees-of-freedom (i.e., 2 in-plane rotations and 3 translations) and with reduced integration.

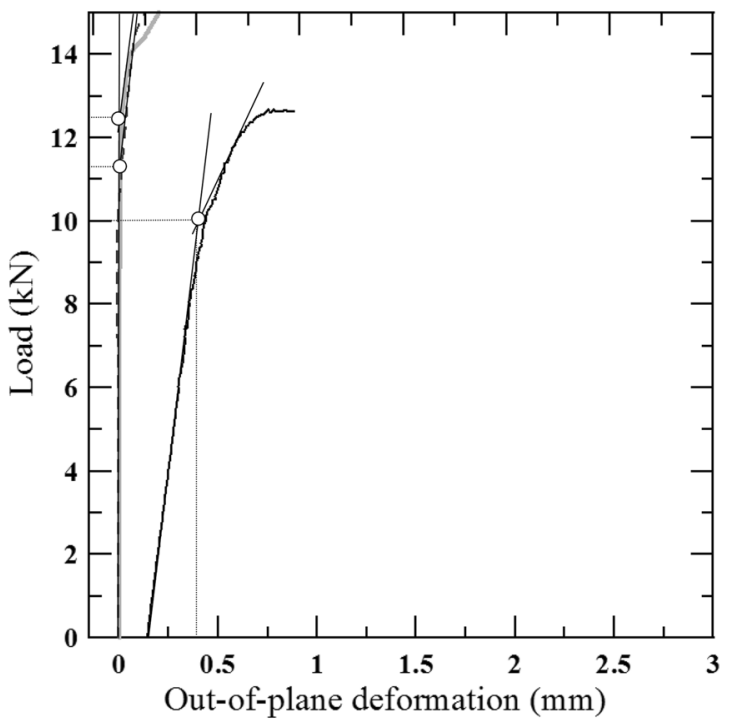

(b) specimen 8
It may be noted here that, in absence of the actual initial imperfections coupled with complexities of the boundary conditions and applied load pattern, the purpose of this numerical simulation is to get an indicative idea of buckling behavior for better understanding of the experimental results.

\subsection{Method of analysis}

Load deformation curve To get an understanding about the experimentally obtained load deformation curves, nonlinear load deformation response was obtained by employing Riks method in the framework of ABAQUS. In Riks method, the load magnitude is used as an additional unknown in addition to displacements and therefore it employs the "arc length" along the static equilibrium path in load-displacement space as another quantity for monitoring the progression of solution. The exact post-buckling case could not be analyzed directly using Riks method due to discontinuity in response at buckling point. Therefore, instead of bifurcation response, the problem is usually modified as a continuous response analysis by introducing an initial imperfection either in the form of loading or geometry so that there is some response before the critical load is reached. Since initial imperfections were not measures, this study employed perturbations in loads to introduce initial imperfections in the modeling. An out-of-plane concentrated load of very small magnitude with a ratio of order $10^{-3}$ to the expected buckling load was applied at the center of the 
Table 3 Effect of size and position of cut-outs on experimental buckling load

\begin{tabular}{lllll}
\hline $\begin{array}{l}\text { Speci- } \\
\text { men no. }\end{array}$ & Aspect ratio & $\begin{array}{l}\text { Cut-out } \\
\text { size } d / b\end{array}$ & $\begin{array}{l}\text { Cut-out number } \\
\text { and position }\end{array}$ & $\begin{array}{l}\text { Buckling } \\
\text { load }(\mathrm{kN})\end{array}$ \\
\hline 1 & 0.838 & 0 & 0 & 3.9 \\
2 & 0.838 & 0.2 & 1 at centre & 3.4 \\
3 & 0.838 & 0.5 & 1 at centre & 2.3 \\
4 & 1.892 & 0 & 0 & 7.8 \\
5 & 1.892 & 0.2 & 1 at centre & 7.8 \\
6 & 1.892 & 0.2 & Right figure & 7.6 \\
7 & 1.892 & 0.5 & 1 at centre & 6.3 \\
8 & 2.885 & 0 & 0 & 10.0 \\
\hline
\end{tabular}

plate (or near the cut-out in plate with cut-outs) as the dead load prior to the Riks step to initiate the instability.

Elastic buckling load For a plate model with $N$ number of degrees of freedom and subjected to a load pattern
$Q^{N}$, the elastic buckling load along with corresponding mode shape in ABAQUS are determined by solving the following governing equilibrium equation

$K^{N N} v^{N}=0$

where $K^{N N}$ is the tangent stiffness matrix under applied loads and $v^{N}$ is the displacement vector of the plate. Now if $\lambda$ is such a multiplier proportional to the load that if Eq. (2) is solved for the loads $\lambda Q^{N}$, the tangent stiffness matrix $K^{N N}$ becomes singular and the problem has nontrivial solutions. Smallest of these corresponds to the lowest mode of buckling of the plate and likewise the higher loads corresponds to higher modes. For an incremental loading pattern $\lambda Q^{N}$, the stiffness matrix consists of two parts as follows:

$K^{N N}=K_{0}^{N N}+\lambda K_{\sigma}^{N N}$
Table 4 Comparison of experimentally obtained and numerically simulated buckling loads

\begin{tabular}{|c|c|c|c|c|c|c|}
\hline \multirow{3}{*}{$\begin{array}{l}\text { Speci- } \\
\text { men no. }\end{array}$} & \multirow{3}{*}{$\begin{array}{l}\text { Simulation }(\mathrm{kN})(\mathrm{A}) \\
\text { Eigenvalue analysis }\end{array}$} & \multicolumn{2}{|c|}{ Simulation $(\mathrm{kN})(\mathrm{B})$} & \multirow{3}{*}{$\begin{array}{l}\text { Experi- } \\
\text { mental } \\
(\mathrm{kN})\end{array}$} & \multirow{2}{*}{\multicolumn{2}{|c|}{$\frac{\% \text { Deviation }}{(\text { Sim.-Exp. }) \times 100 / \text { Sim. }}$}} \\
\hline & & \multirow{2}{*}{$\begin{array}{l}\text { Eigenvalue } \\
\text { analysis }\end{array}$} & \multirow[t]{2}{*}{ Riks method } & & & \\
\hline & & & & & Eigenvalue & Riks method \\
\hline 1 & 5.73 & 4.72 & 4.3 & 3.9 & 17.37 & 9.3 \\
\hline 2 & 4.93 & 4.09 & 4.1 & 3.4 & 16.87 & 17.07 \\
\hline 3 & 4.02 & 3.62 & 3.1 & 2.3 & 36.46 & 25.8 \\
\hline 4 & 12.73 & 8.87 & 8.4 & 7.8 & 12.06 & 7.14 \\
\hline 5 & 13.68 & 8.2 & 7.0 & 7.8 & 4.88 & 11.43 \\
\hline 6 & 12.44 & 8.64 & 7.9 & 7.6 & 12.03 & 3.8 \\
\hline 7 & 15.78 & 6.87 & 6.1 & 6.3 & 8.3 & 3.28 \\
\hline 8 & 19.3 & 13.45 & 11.2 & 10.0 & 25.65 & 10.71 \\
\hline
\end{tabular}

(A) In-plane restraints not considered

(B) Boundary conditions according to Table 2
Fig. 8 a Schematic diagram of buckling mode shapes and b close-up view of a loaded specimen with buckled shape are shown

Loading direction

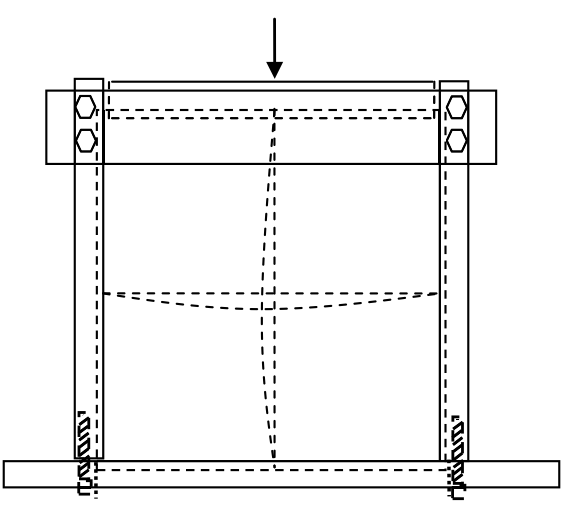

(a)

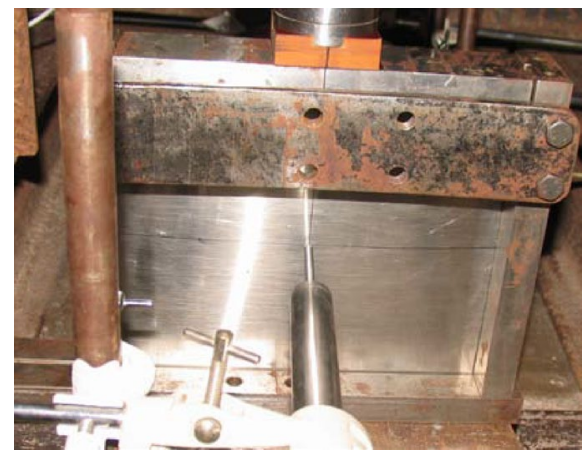

(b) 


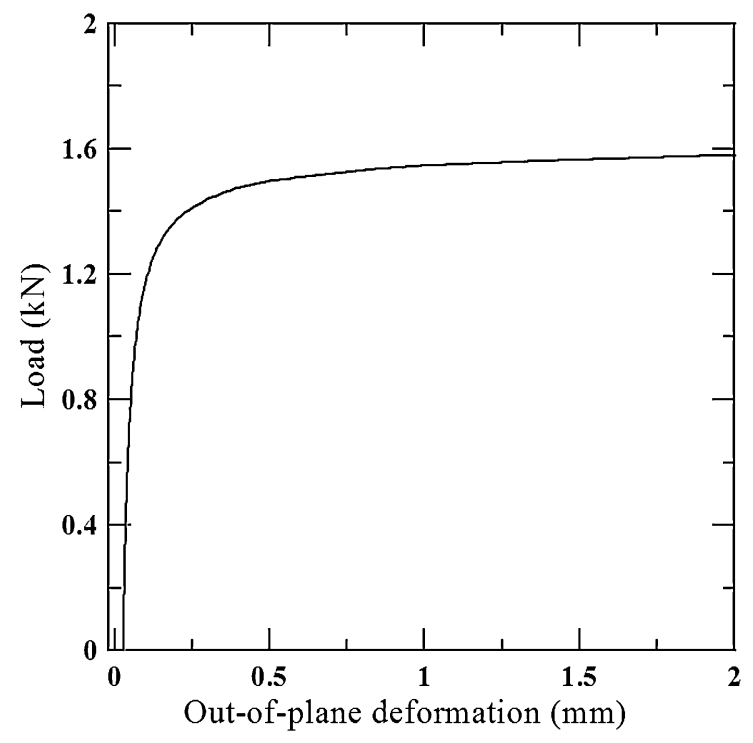

(a)

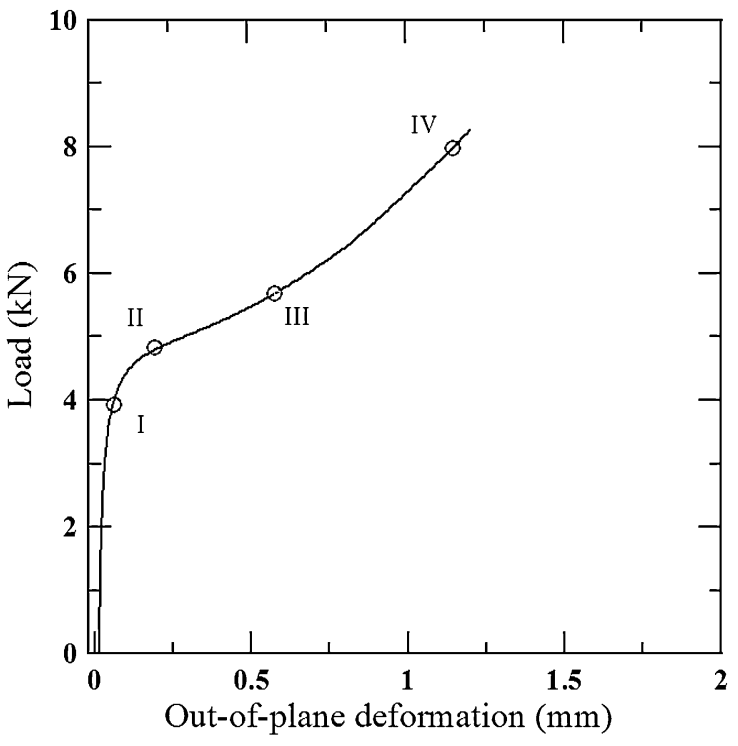

(b)

Fig. 9 Numerically simulated load versus out-of-plane deformation plots are shown: a plate with unsupported unloaded edges $\mathbf{b}$ plate with supported unloaded edges

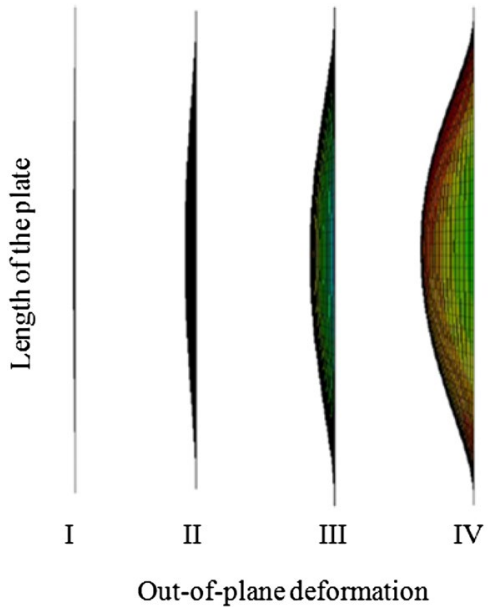

Fig. 10 Numerically simulated deformation patterns of plate with supported unloaded edges at various stages corresponding to Fig. $9 \mathrm{~b}$ are shown

where $K_{0}^{N N}$ corresponds to no-stress state and $\lambda K_{\sigma}^{N N}$ corresponds to incremental load $\lambda Q^{N}\left(\lambda K_{\sigma}^{N N}\right)$. Equation (3) was then substituted in Eq. (2) and solved for $\lambda$. The critical or buckling load for the plate is obtained as the multiplication of minimum of $\lambda$ value and the applied load $Q^{N}$. The corresponding displacement vector $v^{N}$ provided the buckling mode shape. It is to be noted that this displacement vector $v^{N}$ is normalized vector and not the the actual one.

\section{Interpretation of experimental results}

Figures 4, 5, 6 and 7 show a comparison of the load deformation response obtained by both experiment and numerical simulation for all the specimens. The plate in the present study was well supported at the unloaded edges and therefore, the load versus out-of-plane deformation response of the plate showed significant strength after the first stiffness degradation point unlike the plate with unsupported unloaded edges, which behaved more like a column and did not show any post buckling strength (see Fig. 9). However, the first stiffness degradation point on the load deformation curve still can be justified as the buckling load by looking at the deformation pattern of the plate at various load increments (Fig. 10). It is clear from Figs. 9 and 10 that as soon as the load deformation curve reached its first stiffness degradation point, the plate lost its stability and deformed significantly compared to the deformations before the first stiffness degradation point. One may notice from Figs. 4, 5, 6 and 7 that, although the pattern of load-deformation responses from experiments and simulations are showing almost similar trend, the numerically obtained load-deformation curves are showing higher initial stiffness compared to the corresponding experimentally obtained curves. This discrepancy in the load-deformation curves can be attributed to the initial imperfections of the plate and the complexity of the boundary conditions that were not captured in the 


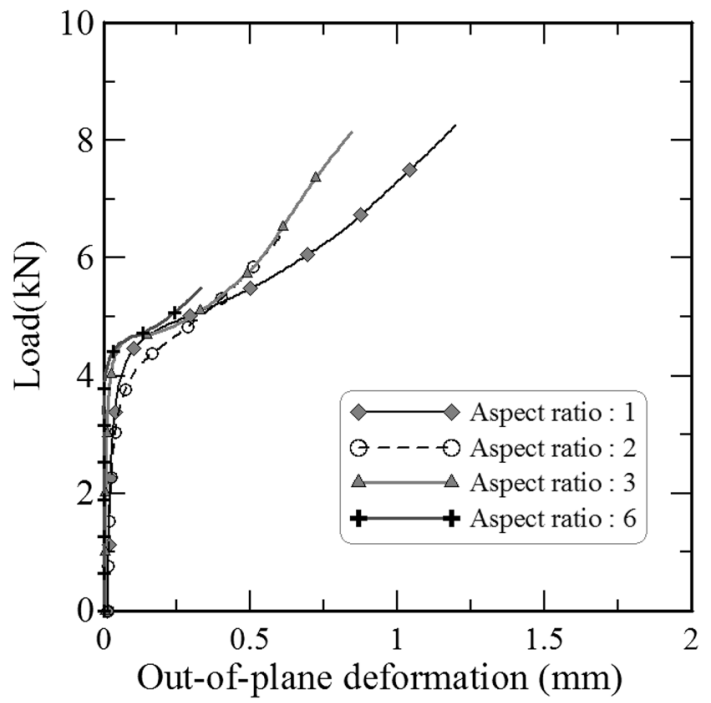

Fig. 11 Comparison of numerically simulated load versus out-ofplane deformation (at the point of maximum deformation) curves for different aspect ratios is shown

simulation models. Further, one may notice from Figs. 4, 5, 6 and 7 that the load-deformation responses were almost similar in both full edge and partial edge loading cases except a slight deviation for specimen with aspect ratio (nearly equal to) 3 . This is because of higher reduction in loading width (16\%) for this aspect ratio. Also, plate with higher aspect ratio showed more stiffness in post buckling range compared to the plate with lower aspect ratio, and therefore, a very gentle slope change in pre-buckling and post buckling range of Fig. $7 \mathrm{~b}$ (aspect ratio nearly 3 ) was observed. To illustrate this fact more clearly, numerically obtained load deformation curves for plate with different aspect ratios were compared as shown in Fig. 11. This clearly strengthens the above mentioned fact. The reason behind this was perhaps the increased length of supported side edges with the increased aspect ratio of the plate. As mentioned earlier, the point where the slope of the load-deformation curve decreases is stated as the buckling load.

To see how the elastic buckling load compared with experimental data, an eigenvalue elastic buckling analysis with the same experimental boundary conditions, was also carried out (using Abaqus [1]). Table 4 shows the comparison of experimental and simulation results (by both Riks method and eigenvalue analysis). In Table 4, the values reported for the numerical simulation are for the loading over the width of the plate specimen in contact of top primary supporting plate only to represent the actual test scenario. It can be observed that, in general, the experimental results showed a good agreement with numerical simulations (both Riks method and eigenvalue analysis) in buckling load with the simulation results showing higher values of buckling load. It can also be noted that the eigenvalue elastic buckling analysis results showed a slightly higher buckling load than the buckling load obtained by Riks method. This may be due to the difference in the way the analysis was performed and buckling loads were estimated. The percentage deviation of experimental and simulation results are also shown in Table 4, wherein this calculation was performed using the simulation results of Riks method. It may be noted that except for specimen 3 , percentage deviation between the experimental and simulation values were within $17 \%$. Table 4 also shows eigenvalue analysis results for numerical model with no in-plane restraints but with identical loading and other boundary conditions. It was observed that the in-plane restraints on plate edges as considered in this study lowered the buckling resistance of the plates. The possible reason for this reduction of buckling load was the difference in pre-buckling in-plane stress distributions in the plates with and without restraints.

\section{Conclusions}

In this study, the influence of size and location of a circular cut-out on the buckling load of simply supported plates with in-plane boundary conditions was investigated experimentally using solid and perforated stainless steel plates with different aspect ratios and edges partially restrained for in-plane movements. Later finite element analyses (eigenvalue elastic buckling and Riks method) were conducted wherein the numerical model closely represented the experimental setup to gain better understanding of the experimentally obtained results. Major findings of this study are listed as follows:

- It was observed from the experimental results that the size and position of the circular cut-out in a plate specimen with in-plane restraints may influence its buckling behavior depending upon the aspect ratio of the plate specimen (i.e., mode of buckling). For aspect ratio close to unity, the presence of cut-out at the center decreases the buckling load of a plate 
specimen. Moreover, as the cut-out size increases, the buckling load reduces. Since, for aspect ratio 1 , the plate buckled in a single half wave, the presence of cut-out at the middle made the plate susceptible to larger out-of-plane deformations, thereby reducing the buckling load.

- A central circular cut-out of size $d / b=0.2$ however does not influence the buckling load of a plate with aspect ratio close to 2 . For a larger cut-out $(d / b=0.5)$ or a cut-out away from the center (in the loading direction), the load however reduces. This is because, the plate with in-plane restraints buckles in the second mode in the loading direction (i.e., two halfwaves). Hence, a larger size of cut-out in the center or moving the cutout away from the center affects the stiffness of the buckling resistance providing zone of the plate specimen.

- The buckling loads obtained from the experiments conducted matched reasonably well with the corresponding simulation results by both Riks method and eigenvalue analysis. In addition, it was found from the analysis that the in-plane transverse restraints on the plate edges reduced the buckling load of the plate.

- Finally, it was noticed that the experimentally obtained load-deformation curves differed noticeably from the simulated ones. This difference in the load-deformation curves can be attributed to the initial imperfections coupled with complexities of the boundary conditions and applied load pattern in the experiments, which could not be accounted accurately in the simulations.

Acknowledgements The assistance of Dr. K. K. Bajpai, Principal Scientific Officer, and other staff of the Structural Engineering Laboratory, Department of Civil Engineering, IIT Kanpur, in conducting the experiments is greatly appreciated.

\section{Compliance with ethical standards}

Conflict of interest On behalf of all authors, the corresponding author states that there is no conflict of interest.

\section{References}

1. Abaqus (2018) Abaqus/CAE user's manual, version 6.10. Abaqus Inc., USA

2. Arbocz J, Weller T (1998) Buckling experiments, basic concepts, columns, beams and plates, vol 1. John Wiley \& Sons
3. Bambach M, Rasmussen K (2004) Experimental techniques for testing unstiffened plates in compression and bending. Exp Mech 44:91-96. https://doi.org/10.1007/BF02427982

4. Bedair OK (1997) Influence of in-plane restraint on the buckling behaviour of plates under uniform compression, shear and in-plane bending. Comput Methods Appl Mech Eng 148:1-10. https://doi.org/10.1016/S0045-7825(97)00035-2

5. Bedair OK, Sherbourne AN (1994) On the stability of plates under combined compression and in-plane bending. Comput Struct 53:1453-1464. https://doi.org/10.1016/0045-7949(94)90410-3

6. Bedair OK, Sherbourne AN (1995) The elastic stability of partiallyrestrained plates under compression and in-plane bending. J Constr Steel Res 35:339-360. https://doi.org/10.1016/0143974X(94)00052-J

7. Bradfield CD (1980) Tests on plates loaded in in-plane compression. J Constr Steel Res 1:27-37. https://doi.org/10.1016/0143974X(80)90006-1

8. Chou SM, Rhodes J (1997) Review and compilation of experimental results on thin-walled structures. Comput Struct 65:4767. https://doi.org/10.1016/S0045-7949(96)00337-9

9. Dietrich L, Kawahara W, Phillips A (1978) An experimental study of plastic buckling of a simply supported plate under edge thrusts. Acta Mech 29:257-267. https://doi.org/10.1007/BF011 76641

10. El-Sawy KM, Nazmy AS (2001) Effect of aspect ratio on the elastic buckling of uniaxially loaded plates with eccentric holes. Thin-Walled Struct 39:983-998. https://doi.org/10.1016/S0263 -8231(01)00040-4

11. Featherston CA, Eaton MJ, Holfrod KM (2008) Imperfection sensitivity in plates-an assessment using dic. SEM XI international congress \& exposition on experimental \& applied mechanics

12. Maiorana E, Pellegrino C, Modena C (2009) Elastic stability of plates with circular and rectangular holes subjected to axial compression and bending moment. Thin-Walled Struct 47:241255. https://doi.org/10.1016/j.tws.2008.08.003

13. Mínguez JM (1986) An experimental investigation of how accurate, simply supported boundary conditions can be achieved in compression testing of panels. Exp Mech 26(3):238-244. https ://doi.org/10.1007/BF02320048

14. Moxham KE (1971) Buckling tests on individual welded steel plates in compression. Report no., University of Cambidge, Department of Engineering

15. Prajapat K (2011) Analytical and experimental study of buckling of plates considering in-plane boundary conditions. M.S. thesis, Indian Institute of Technology Kanpur, Indian Institute of Technology Kanpur

16. Prajapat K, Ray-Chaudhuri S, Kumar A (2015) Effect of in-plane boundary conditions on elastic buckling behavior of solid and perforated plates. Thin-Walled Struct 90:171-181. https://doi. org/10.1016/j.tws.2014.12.015

17. Roberts TM, Azizian ZG (1984) Strength of perforated plates subjected to in-plane loading. Thin-Walled Struct 2:153-164. https ://doi.org/10.1016/0263-8231(84)90009-0

18. Sabir AB, Chow FY (1986) Elastic buckling of plates containing eccentrically located circular holes. Thin-Walled Struct 4:135149. https://doi.org/10.1016/0263-8231(86)90020-0

Publisher's Note Springer Nature remains neutral with regard to jurisdictional claims in published maps and institutional affiliations. 